\title{
Reviewing imaging modalities for the assessment of plaque erosion
}

\author{
Carlos Collet $^{\mathrm{a}, 1}$, Edoardo Conte ${ }^{\mathrm{b}, \mathrm{c}, 1}$, Saima Mushtaq ${ }^{\mathrm{b}, \mathrm{c}}$, Sofie Brouwers ${ }^{\mathrm{a}, \mathrm{d}}$, Toshiro Shinke ${ }^{\mathrm{e}}$, \\ Ahmet Umit Coskun ${ }^{\mathrm{f}}$, Zhongyue Pu ${ }^{\mathrm{g}}$, Diaa Hakim ${ }^{\mathrm{g}}$, Peter Howard Stone ${ }^{\mathrm{f}}$, Daniele Andreini ${ }^{\text {b,c, }}$ \\ ${ }^{\text {a }}$ Cardiovascular Center Aalst, OLV Hospital, Aalst, Belgium \\ ${ }^{\mathrm{b}}$ Centro Cardiologico Monzino, IRCCS, Milan, Italy \\ ${ }^{\mathrm{c}}$ Department of Clinical Sciences and Community Health, Cardiovascular Section, University of Milan, Milan, Italy \\ ${ }^{\mathrm{d}}$ Experimental Pharmacology, Faculty of Medicine and Pharmacy, Vrije Universiteit Brussel, Brussels, Belgium \\ ${ }^{\mathrm{e}}$ Division of Cardiology, Department of Medicine, Showa University School of Medicine, Japan \\ ${ }^{\mathrm{f}}$ Northeastern University, Boston, MA, USA \\ ${ }^{\mathrm{g}}$ Cardiovascular Division, Brigham \& Women's Hospital, Harvard Medical School, Boston, MA, USA
}

\section{A R T I C L E I N F O}

\section{Keywords:}

Plaque erosion

Optical computed tomography

Intravascular ultrasound

Coronary computed tomography

\begin{abstract}
A B S T R A C T
Plaque rupture followed by intracoronary thrombus formation is recognized as the most common pathophysiological mechanism in acute coronary syndromes (ACS). The second most common underlying substrate for ACS is plaque erosion whose hallmark is thrombus formation without cap disruption. Invasive and non-invasive methods have emerged as a promising tool for evaluation of plaque features that either predict or detect plaque erosion. Optical coherence tomography (OCT), high-definition intravascular ultrasound (IVUS), nearinfrared spectroscopy (NIRS), and near-infrared autofluorescence (NIRF) have been used to study plaque erosion. The detection of plaque erosion in the clinical setting, mainly facilitated by OCT, has shed light upon the complex pathophysiology underlying ACS not related to plaque rupture. Coronary computed tomography angiography (CCTA), which is to date the most commonly used non-invasive technique for coronary plaque evaluation, may also have a role in the evaluation of patients predisposed to erosion. Also, computational models enabling quantification of endothelial shear stress may pave the way to new research in coronary plaque pathophysiology. This review focuses on the recent imaging techniques for the evaluation of plaque erosion including invasive and non-invasive assessment.
\end{abstract}

\section{Introduction}

Acute coronary syndromes (ACS) occur due to a sudden decrease in myocardial blood flow following a thrombotic event. Plaque rupture with subsequent thrombus formation is the main pathophysiological mechanism, responsible of $65-75 \%$ of ACS cases. Plaque rupture usually occurs at a lipid-rich inflamed plaque, often with a necrotic core, covered with a thin fibrous cap (thin-cap fibroatheroma, TCFA) [1]. TCFAs have been largely characterized by autoptic studies and by invasive and non-invasive imaging [2,3]. The second most common underlying substrate for ACS is plaque erosion, causing $25-35 \%$ of ACS cases [1]. The hallmark of plaque erosion is thrombus formation without cap disruption. Eroded plaques contain mainly smooth muscle cells and proteoglycan-collagen matrix, without the presence of intimal endothelial cells [1]. Plaque erosion is more frequent in younger smoking females, although recent evidence suggests that also younger smoking males are susceptible [4]. Thrombosis occurs when blood comes into direct contact with intimal surface lacking endothelial cells, nonetheless, the precise mechanisms leading to plaque erosion remain somewhat elusive [5].

Several imaging methods have been used for the evaluation of plaque erosion (Table 1). Optical coherence tomography (OCT), an intravascular method based on light with high spatial resolution, has facilitated the detection of plaque erosion in the clinical setting [4]. Other methods, such as high-definition intravascular ultrasound (IVUS), near-infrared spectroscopy (NIRS), near-infrared autofluorescence (NIRF) and coronary computed tomography angiography (CCTA), have been also proposed to assess plaque erosion. These imaging methods have increased

\footnotetext{
* Corresponding author. FESC, FSCCT, Via C. Parea 4, 20138 Milan, Italy.

E-mail address: daniele.andreini@ccfm.it (D. Andreini).

1 equally contributed to the manuscript.
} 
Table 1

Imaging modalities for atherosclerosis evaluation.

\begin{tabular}{llll}
\hline & \multicolumn{4}{l}{ Non-invasive imaging } \\
\cline { 2 - 4 } & CCTA & PET & MRI \\
\hline Contrast needed & Yes & Yes & No \\
Radiation burden & + & ++ & - \\
Spatial resolution & $0.2-0.5 \mathrm{~mm}$ & $5 \mathrm{~mm}$ & $0.5-1 \mathrm{~mm}$ \\
Prognostic value & +++ & ++ & - \\
Time of data acquisition & $1-5 \mathrm{~min}$ & $30-60 \mathrm{~min}$ & $30-60 \mathrm{~min}$ \\
Availability & +++ & + & + \\
\hline & Invasive imaging & & \\
& OCT & IVUS & NIRS \\
\hline Spatial resolution & $15-20 \mu \mathrm{m}$ & $20-40 \mathrm{~mm}$ & - \\
Tissue penetration & + & ++ & ++ \\
Contrast needed & Yes & No & No \\
Availability & ++ & ++ & + \\
\hline
\end{tabular}

CCTA: coronary computed tomography; PET: positron emission toography; MRI: magnetic resonance imaging; OCT: optical computed tomography; IVUS-vh: intravascular ultrasound-virtual histology; NIRS: near infrared spectroscopy.

our understanding of the complex pathophysiology associated with plaque erosion. Plaque erosion is getting more attention due to its prevalence among ACS patients and the increasing use of invasive imaging methods [5].

Invasive and non-invasive methods have emerged as promising tools for assessing plaque features that either predict or detect plaque erosion. This review focuses on the recent imaging techniques for the evaluation of plaque erosion including both invasive and non-invasive assessment.

\section{Invasive imaging modalities}

\subsection{Optical coherence tomography}

Optical coherence tomography is an intracoronary diagnostic technique that provides detailed imaging of coronary vessels. Since its introduction, more than two decades ago, OCT has been useful in the assessment of atherosclerotic lesions, characterization of plaque components and as guidance percutaneous coronary interventions [6]. OCT uses infrared light (1.3 mm wavelength), which confers high spatial resolution in the range of $15-20 \mu \mathrm{m}$; however, with limited tissue penetration $(1-2 \mathrm{~mm})$ [6]. OCT visualizes the luminal surface of the vessel and the microstructure of atherosclerotic plaque such as fibrous cap, thrombus, and calcifications [6]. These features make OCT the most suited diagnostic technique to assess plaque erosion in the clinical setting.

Pathologically, plaque erosion is defined as a loss of endothelial lining with lacerations of the superficial intimal layers in the absence of cap rupture [4]. Nevertheless, OCT is unable to detect endothelial cells sloughing. Therefore, plaque erosion is defined and categorized at OCT according to the absence of fibrous cap disruption and presence of thrombus. OCT-defined plaque erosion is then divided into definite erosion and probable erosion. Definite erosion is identified by the presence of a luminal thrombus overlying an intact plaque while probable erosion is defined as (i) luminal surface irregularity at the culprit lesion in the absence of thrombus; or (ii) attenuation of underlying plaque by thrombus without superficial lipid or calcification immediately proximal or distal to the site of the thrombus (Fig. 1) [4]. This is in contrast with the pathological definition of erosion, which requires the presence of attached thrombus [7]. Plaques complicated by erosion tend

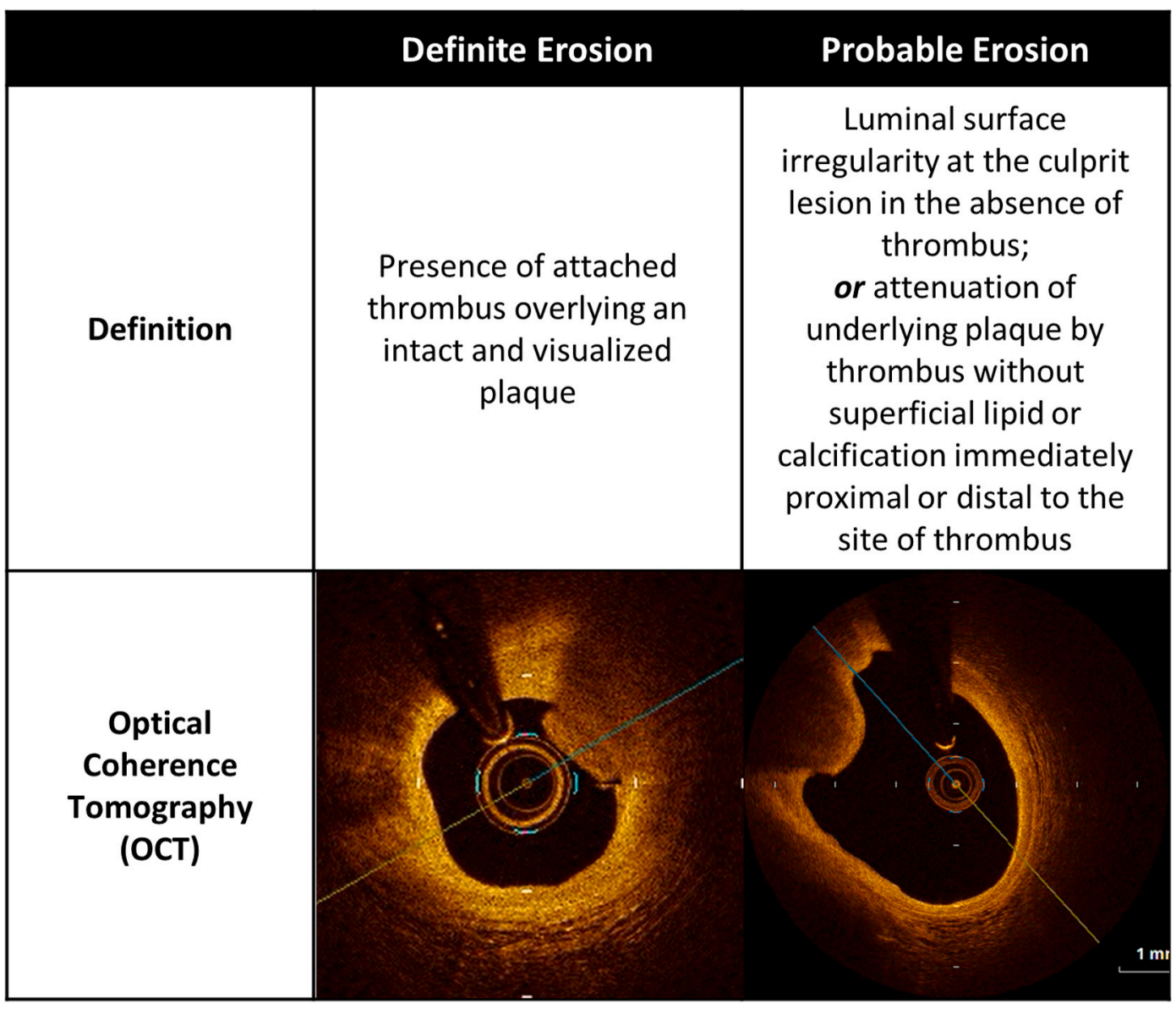

Fig. 1. OCT definition of plaque erosion. 
to be matrix-rich, lipid-poor and usually lack prominent macrophage collections, unlike plaques that rupture, which characteristically have thin fibrous caps, large lipid pools and abundant foam cells. Thrombi that complicate superficial erosion seem more platelet-rich than the fibrinous clots precipitated by plaque rupture [8].

In contemporary cohorts, erosion appears to account for as high as one-third of ACS [4,9]. Plaque erosion has been shown to be more prevalent in non-ST elevation myocardial infarction than in patients presenting with ST-elevation myocardial infarction [4,9]. The largest prospective cohort to date included 822 ST-elevation myocardial infarction patients with systematic OCT pre-intervention. Using established diagnostic criteria, 209 of the patients had plaque erosion (25.4\%). Plaque erosion was more frequent in women $<50$ years when compared with those $\geq 50$ years of age. Patients with plaque erosion were more frequently smokers but had fewer other coronary risk factors (e.g. dyslipidemia, hypertension, chronic kidney disease, and diabetes mellitus) than those with plaque rupture. The left anterior descending artery is the most prevalent location for erosion [10]. Furthermore, plaque erosion lesions had a lower percentage of stenosis as compared with plaque ruptures, and had a lower prevalence of lipid-rich plaque, less lipid content, and less calcification, and more were frequently located near bifurcations as compared with plaque ruptures [810]. Fig. 2 shows the differential clinical, angiographic and OCT features between plaque erosion and rupture.

The recognition of plaque erosion as a distinct clinical entity prompted the exploration of alternative treatment options other than percutaneous coronary interventions (PCI). The EROSION study included patients with ACS, OCT-defined plaque erosion and residual diameter stenosis of less than $70 \%$. These patients were treated with anti-thrombotic therapy (aspirin and ticagrelor) without stenting [11]. OCT was repeated at 1 month, in 47 out of 60 patients, a reduction of more than $50 \%$ in thrombotic burden was observed, and in 22 patients no visible thrombus was observed [11]. At 1 year, 49 patients underwent subsequent OCT imaging. Almost half of the patients (46.9\%) had no residual thrombus at 1 year [12]. Based on the results of this proof of concept study, it is suggested that selected patients presenting with plaque erosion could be managed conservatively without stenting. This finding requires confirmation in larger prospective clinical trials.

\subsection{High-definition IVUS}

High-definition intravascular ultrasound (IVUS) represents the current state of the art in IVUS imaging with an axial resolution of approximately $20-40 \mu \mathrm{m}$, faster cath-lab pullback speed up to $10 \mathrm{~mm} / \mathrm{s}$, and rapid image acquisition of 60 frames/sec [6]. The main advantage of IVUS over OCT is its tissue penetration and the fact that it does not requires contrast injection for image acquisition. High-definition IVUS facilitates analysis of the luminal surface and, therefore, could be used for detection of plaque erosion. Case series support the potential value of high definition IVUS for the evaluation of plaque erosion [13]. The

\begin{tabular}{|c|c|c|}
\hline & Plaque Rupture & Plaque Erosion \\
\hline $\begin{array}{c}\text { Estimated } \\
\text { incidence ACS }\end{array}$ & $\begin{array}{c}67 \% \\
\text { (Predominant in STEMI) }\end{array}$ & $\begin{array}{c}25 \% \\
\text { (NSTEMI>STEMI) }\end{array}$ \\
\hline Clinical Profile & $\begin{array}{c}\text { Older } \\
\text { Traditional risk factors } \\
\text { (dyslipidemia, hypertension, } \\
\text { diabetes) }\end{array}$ & $\begin{array}{l}\text { Younger } \\
\text { Women } \\
\text { Smoker } \\
\text { Lower LDL }\end{array}$ \\
\hline $\begin{array}{l}\text { Angiographic } \\
\text { characteristics }\end{array}$ & $\begin{array}{c}\text { Higher anatomical complexity } \\
\text { Smaller lumen } \\
\text { Occlusive thrombus }\end{array}$ & $\begin{array}{c}\text { Predominant LAD } \\
\text { Lower anatomical complexity } \\
\text { Larger lumen } \\
\text { Non-occlusive thrombus }\end{array}$ \\
\hline $\begin{array}{l}\text { Underlying } \\
\text { Plaque }\end{array}$ & $\begin{array}{l}\text { Lipid plaque } \\
\text { TCFA } \\
\text { Red thrombus }\end{array}$ & $\begin{array}{l}\text { Fibrous plaque } \\
\text { White thrombus }\end{array}$ \\
\hline $\begin{array}{l}\text { Optical } \\
\text { Coherence } \\
\text { Tomography } \\
\text { (OCT) }\end{array}$ & & \\
\hline
\end{tabular}

Fig. 2. Clinical, angiographic and optical coherence tomography characteristics between plaque erosion and rupture. 
presence of a normal vessel wall and minor intimal irregularities with or without thrombus suggests the diagnosis of plaque erosion (Fig. 3). In the presence of fibrotic or lipid plaques, the finding of surface irregularities or layered images without cap rupture is also suggestive of plaque erosion. However, it must be recognized that the diagnosis of plaque erosion with IVUS remains challenging. IVUS lacks the resolution to directly image fibrous cap thickness. Howver, IVUS can be particularly helpful in patients suspected to have plaque erosion, mainly in cases of concomitant renal dysfunction where the additional use of contrast medium, for example for OCT image acquisition, should be limited during the invasive procedure to minimize the risk of contrast induced nephropathy.

IVUS have allowed for a better understanding of pathophysiological mechanisms associated with plaque erosion [14]. Studies using both IVUS and OCT have shown that vessels with plaque erosion exhibit negative remodeling in contrast to cases with plaque rupture that typically show positive remodeling [14]. Moreover, lesions with plaque erosion exhibit lower plaque burden compared to lesions with plaque rupture [14]. The addition of virtual histology to IVUS further enabled to differentiate underlying plaque component in patients with plaque erosion and rupture. In cases of erosion, the underneath plaque is predominantly fibrotic with white thrombus. Plaque rupture cases exhibit lipidic plaque and red thrombus $[14,15]$. Lesions with plaque erosion tend to be more eccentric compared to those with plaque rupture. Hence, the finding of an eccentric fibrotic plaque with surface irregularities suggesting white thrombus may further support the diagnosis of plaque erosion by IVUS. Interestingly, the incidence of no-reflow phenomenon and with microvascular damage after PCI has been reported to be lower in cases of plaque erosion compared to plaque rupture [14].

\subsection{Near-infrared spectroscopy (NIRS)}

Near-infrared spectroscopy measures the wavelength-dependent

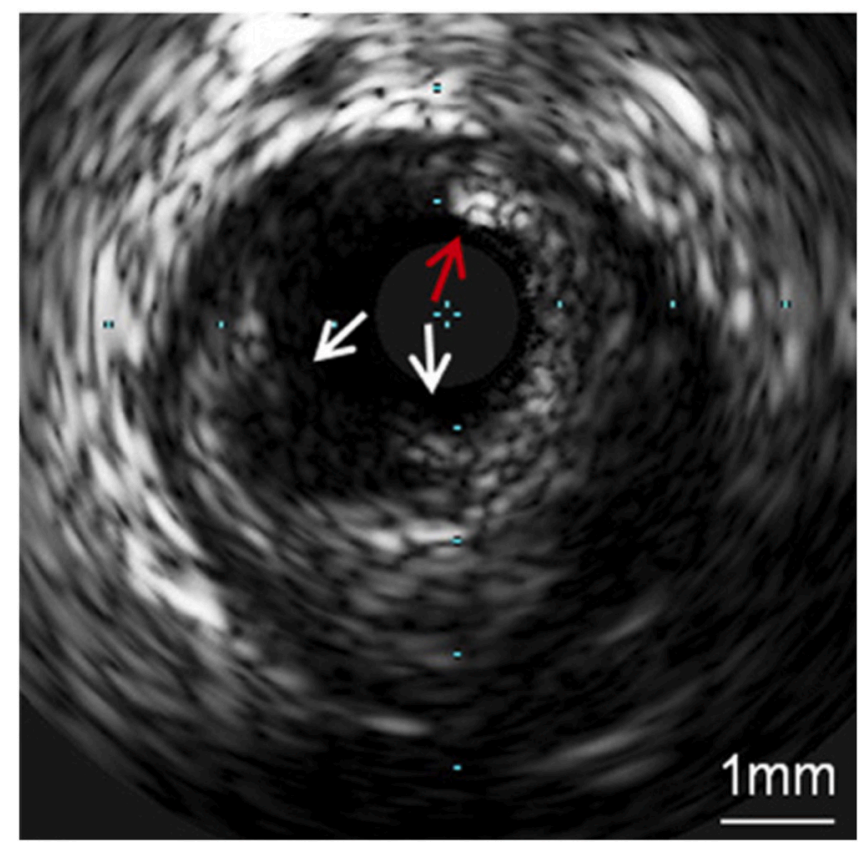

Fig. 3. Plaque erosion visualized using high-definition intravascular ultrasound.

A 37-year-old woman presenting with non-st-segment elevation myocardial infarction. High-definition intravascular ultrasound showed images of a lipid plaque with surface irregularities without cap rupture, suggestive of plaque erosion (yellow dotted arrows). Asterisk indicates wire artifact. Reproduced with permission of Cuesta et al. (JACC: Cardiovascular Interventions Volume 13, Issue 7, April 2020). interaction of electromagnetic radiation with matter [5]. NIRS is uniquely suited for analysis of lipid core plaques in coronary arteries since it can penetrate into blood and several millimeters into the tissue. NIRS provides a specific chemical measure of lipid core plaques, since cholesterol can be distinguished from other tissue constituents such as collagen. For clinical use, NIRS is coupled with an IVUS system with a pullback and rotation unit, similar in size to traditional IVUS catheters [16].

NIRS-IVUS imaging adds to the armamentarium as a diagnostic tool able to detect vulnerable plaques based on the amount of the lipidic core. NIRS-IVUS imaging-derived Lipid Core Burden Index (LCBI) has demonstrated that it is able to identify patients at higher risk for the occurrence of adverse events (i.e., combined endpoint cardiac death, cardiac arrest, non-fatal myocardial infarction, ACS, revascularization by coronary artery bypass grafting or PCI, and readmission to hospital for angina with more than $20 \%$ diameter stenosis progression related and unrelated to the treatment at index procedure) [17]. While the role of NIRS in clinical practice remains to be determined, it has expanded our knowledge in cases of plaque erosion. NIRS has been shown to discriminate OCT-defined erosions into two distinct phenotypes: lesions with high or no detectable LCBI (Fig. 4) [18]. The clinical implication of these findings remains to be elucidated; nonetheless, it can be hypothesized that plaque erosion without a lipidic core may carry better prognosis compared to plaque erosion with a lipid-rich substrate.

\subsection{Near-infrared fluorescence}

Near infrared fluorescence (NIRF) molecular imaging allows for intravascular imaging of biological details in coronary arteries [5]. A catheter combining NIRF and OCT has been validated in vivo. NIRF quantifies plaque inflammation and could enhance the identification of plaques at risk for progression and complication [5]. Moreover, NIRF molecular imaging informs on plaque protease activity and abnormal endothelial permeability [19]. An impaired endothelial barrier function has been implicated as mechanism leading to plaque erosion. In rabbits, plaques that exhibit impaired in vivo endothelial permeability were susceptible to subsequent thrombosis; interestingly, these sites rarely displayed plaque rupture. Superficial erosion and subsequent plaque thrombosis merit further analysis, in particular the assessment of endothelial integrity and its role in atherothrombosis [19]. NIRF may play a role in identifying plaque vulnerable to plaque erosion.

\section{Non-invasive imaging modalities}

\subsection{Coronary compueted tomography angiography (CCTA)}

In the last decade, CCTA clinical applications has been extended as several technological improvements were introduced. Last generation CT scans with up to $0.23 \mathrm{~mm}$ spatial resolution, high gantry rotation speed and whole heart coverage detector, provided further reduction of radiaton dose and contrast medium needed.

In the recent European Society of Cardiology (ESC) guidelines for chronic coronary syndrome (CCS), CCTA received a Class IB indication as the initial test for diagnosing CAD in symptomatic patients [20]. Beyond anatomical identification of obstructive lumen stenosis, recent data support CCTA as the main diagnostic tool for non-invasive evaluation of atherosclerosis itself, having a good accuracy when compared to invasive imaging modalities (i.e. OCT and IVUS) [21-23]. High-risk plaque features identified by CCTA have been correlated with cardiovascular prognosis, and results from the SCOT-HEART trial suggested that the adoption of CCTA as the first step test may result in a reduction of cardiovascular events rate, possibly due to an increased prescription of preventive therapies (aspirin and statin), especially among patients with non-obstructive CAD whose risk would be underestimated by functional tests [24-27].

Beyond coronary artery calcium score (CACS), representing the most enduring and validated tool for prognostic stratification based on non- 

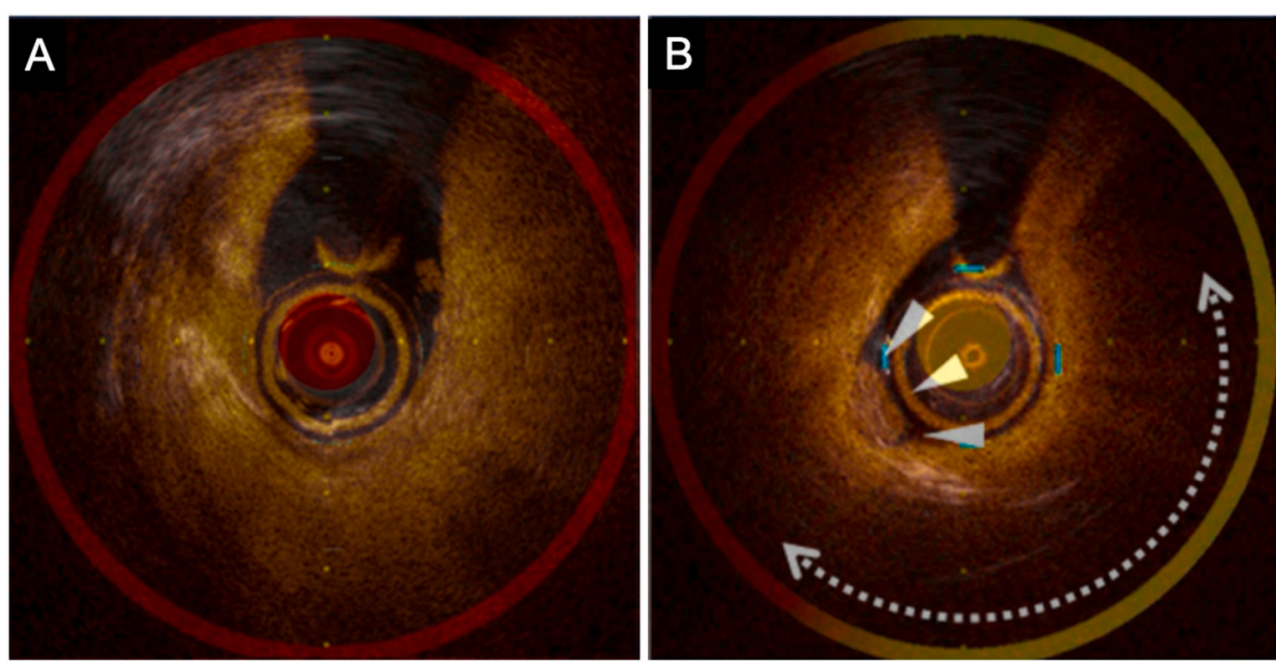

Fig. 4. Near-infrared spectroscopy phenotypes in cases of plaque erosion.

Superimposition of OCT and NIRS-IVUS imaging in two cases of plaque erosion. (A) OCT examination with smooth luminal surface with thrombus overlying a fibrous plaque at the culprit lesion, categorized as OCTerosion. NIRS-IVUS revealed a fibrous plaque without lipid. Max LCBI4mm was 0 (red ring). (B) OCT examination revealed a smooth luminal surface with thrombus (white arrowheads) overlying a lipid-rich plaque characterized by the presence of signal-poor lipid pool (white arc), categorized as OCTerosion. NIRS-IVUS revealed a lipid-rich plaque with echo attenuation. Max lipid core burden index of a 4-mm segment (max LCBI4mm, yellow ring) was 628. Reproduced with permission of Yamaguchi et al. (European Heart Journal - Case Reports (2020) 4, 1-5). invasive evaluation of atherosclerosis by CCTA, several high-risk plaque features could be identified at CCTA, such as positive remodeling, lowattenuation plaque, napkin ring sign and spotty calcification (Fig. 5) [28]. All these features were originally related to invasive identification of rupture-prone TCFA that is characterized by the presence of thin fibrous cup surrounding an inflamed lipid core [29]. However, CCTA cannot make a distinction between a fibrous cap and the underlying necrotic core; in addition it is difficult to differentiate an intra-plaque hemorrhage and a lipid-rich-core. In 2009, Motoyama et al. reported one of the seminal papers in this field suggesting that at 27 months, patients with both positive remodeling (PR) and low-attenuation plaque (LAP) had an adverse event rate of $22 \%$, while those with neither LAP or PR had an adverse event rate of $0.5 \%$, respectively [30]. Plaque erosion is a different mechanism of plaque instability characterized by intact fibrous cap at the time of ACS and occurs more frequently in women and younger patients. In 2011, Ozaki et al. reported the absence of specific CCTA plaque characteristics able to differentiate intact fibrous plaque leading to ACS from stable lesions among 57 culprit lesions evaluated

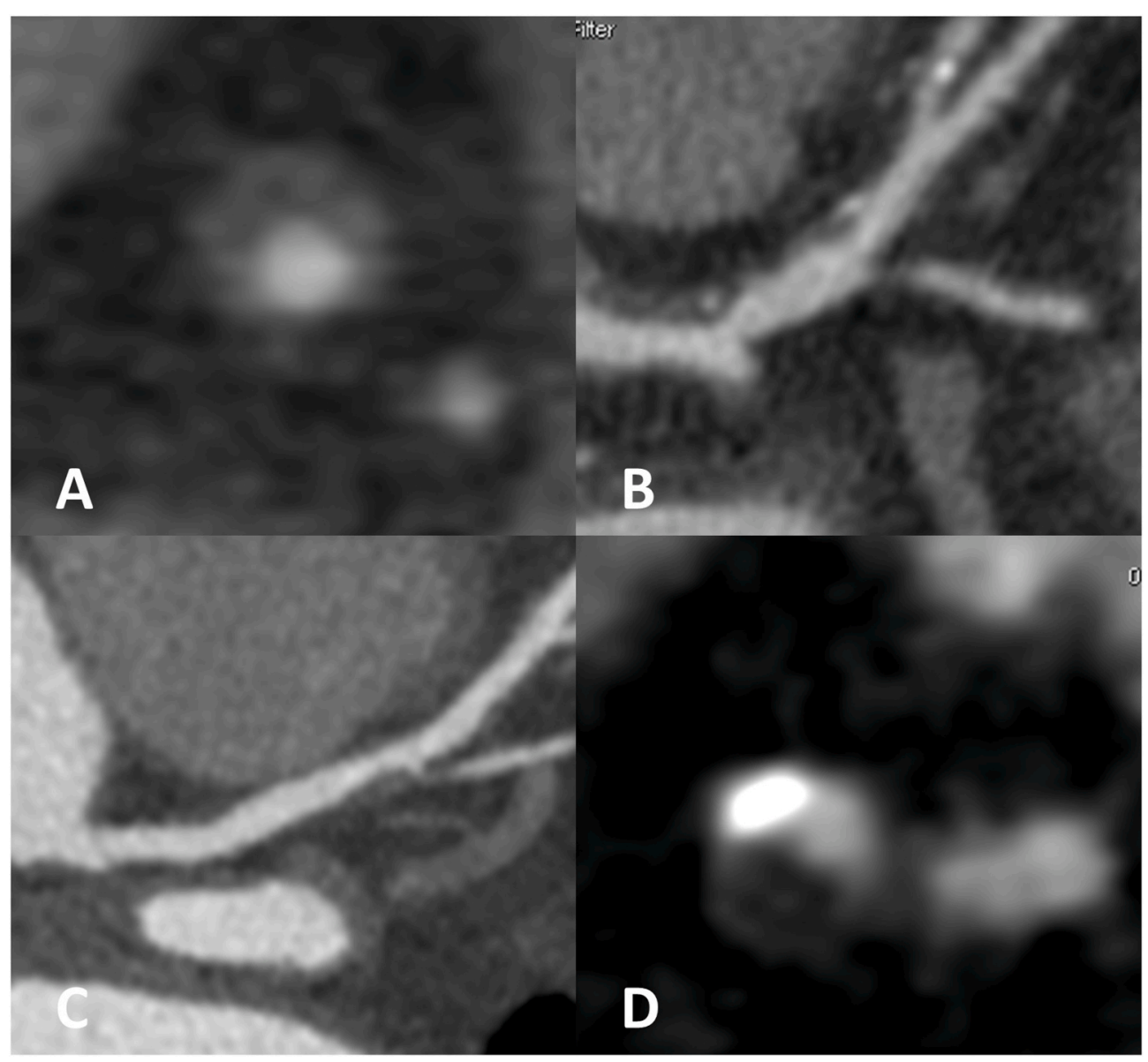

Fig. 5. High risk plaque features at CCTA. (A) High risk plaque features at CCTA: Napkin ring sign defined as the presence of rim-like thin enhancement (no more than $130 \mathrm{HU}$ ) distributed along the outer contour of the vessel and surrounding a fibro-lipidic plaque; (B) spotty calcification defined as any discrete calcification $\leq 3 \mathrm{~mm}$ in length and occupying $\leq 90^{\circ}$ arc when viewed in short axis; (C) remodeling index defined as the ratio between lesion plaque area and reference lumen area; (D) low attenuation plaque defined as the presence of any voxel $<30 \mathrm{HU}$ in a coronary plaque. 
with invasive (OCT) and non-invasive (CCTA) imaging [31]. Of interest, anecdotal reports suggest that erosion-prone plaques could have CCTA adverse plaque features similar to those described for TCFA, such as positive remodeling, low-attenuation plaque and napkin ring sign [32, 33]. Overall, evidence supporting the capability of CCTA to specifically identify erosion-prone plaque are scarce and mostly negative. However, this would not disqualify CCTA as the main tool for non-invasive coronary atherosclerosis evaluation; indeed, even if fine and detailed characterization of plaque subtype (rupture $v s$ erosion prone plaques) does not appear to be achievable, CCTA provides clinicians with several information regarding overall atherosclerosis burden and plaque volume subtypes that are associated with future cardiovascular events [34]. Plaque subtypes volume quantification appears to be related not only to future cardiovascular events, but also to lesion progression into obstructive lesions [35]. The association between high risk plaque features with atherosclerosis progression is consistent with the pathophysiological hypothesis considering plaque progression as a necessary step between stable atherosclerosis and acute clinical event [36].

\subsection{Endothelial shear stress}

Endothelial shear stress (ESS), the tangential force derived by the friction of the flowing blood on the endothelial surface, was recently identified as an important factor in the pathophysiology of plaque erosion [37]. While chronic exposure to low ESS has been associated with endothelial inflammation leading to plaque progression, fibrous cap thinning rendering the plaque more prone to rupture [38], Yamamoto et al. suggested that high ESS/EES gradient (ESSG) at the throat of the plaque may favor the occurrence of plaque erosion [37]. CCTA has been recently described as a promising tool for the evaluation of shear stress, potentially overcoming the need of invasive evaluation (Fig. 6) [39]. Moreover, high-ESS had an incremental value over stenosis severity in the prediction of adverse plaque features presence $[40,41]$. Similar results have been reported by Han et al. in a population of patients that underwent both CCTA and invasive coronary angiography with fractional flow reserve suggesting that ESS was associated with adverse plaque features independent of stenosis severity [42]. However, ESS displayed no incremental benefit for detection of lesions that caused ischemia beyond stenosis severity and atherosclerotic plaque characteristics (APCs) presence. Of interest, the EMERALD study, retrospectively included 66 culprit and 150 non-culprit lesions among 72 patients with documented ACS who underwent CCTA between 1 month and 2 years before index ACS. Here, the addition of ESS on top of adverse plaque features improved the detection of culprit lesion for future ACS [35]. In addition, plaque structural stress, representing the stress located inside an atherosclerotic plaque as a consequence of vessel expansion and stretch induced by exposure to arterial pressure, has been shown to be associated with compositional changes suggestive of increased plaque vulnerability; thus, the interplay between PSS and WSS may have added value in predicting plaque related events [43].

\subsection{Other non-invasive imaging technique}

Non-invasive imaging techniques for coronary plaque evaluation, other than CCTA, include cardiac magnetic resonance (CMR) and positron emission tomography (PET). Preliminary data support the capability of CMR to identify lipid-rich coronary plaque with intra-plaque hemorrhage due to a high T1 weighted signal [44]. Similarly, post-contrast imaging may evidence increased accumulation of Gadolinium into inflamed plaque; however, the low spatial resolution of CMR ( $0.6 \mathrm{~mm}$ with CMR $v s 0.23 \mathrm{~mm}$ in CT) may limit its clinical application that is nowadays used only in dedicated research hospitals [45]. In 2010, Kato et al., reported a diagnostic accuracy of 79\% (CI 95\% 72-86\%) for cardiac MRI in the identification of significative coronary stenosis (more thant $50 \%$ stenosis) $v s$ invasive coronary angiography [46]. More recently, Hosoda et al. suggested that coronary plaque with hyperintesity signal in T1w images were associated with peri-rocedural myocardial infarction. However, even if some data suggest the potential use of MRI for advanced plaque imaging, this type of evaluation is far from a clinical use on a daily basis and no data are available on the possibility to predict plaque erosion. Atherosclerosis inflammatory activity could be evaluated with different PET tracers (i.e. ${ }^{18} \mathrm{~F}$-fluorodeoxyglucose; ${ }^{18} \mathrm{~F}$-sodium fluoride etc); however, some tracer like ${ }^{18} \mathrm{~F}$-fluorodeoxyglucose are well-validated for extracardiac atherosclerosis evaluation but cannot be used for coronary atherosclerosis due to avid myocardium uptake [47]. Moreover, the limited anatomical definition needs hybrid imaging to be implemented with CT or MRI; advanced CT application including artificial intelligence and radiomics has been recently demonstrated to adequately identify event-prone plaque when compared to invasive and functional imaging (PET). However, no data are available regarding the capability of CMR and/or PET to distinguish erosion-prone plaque from other forms of vulnerable atherosclerosis.

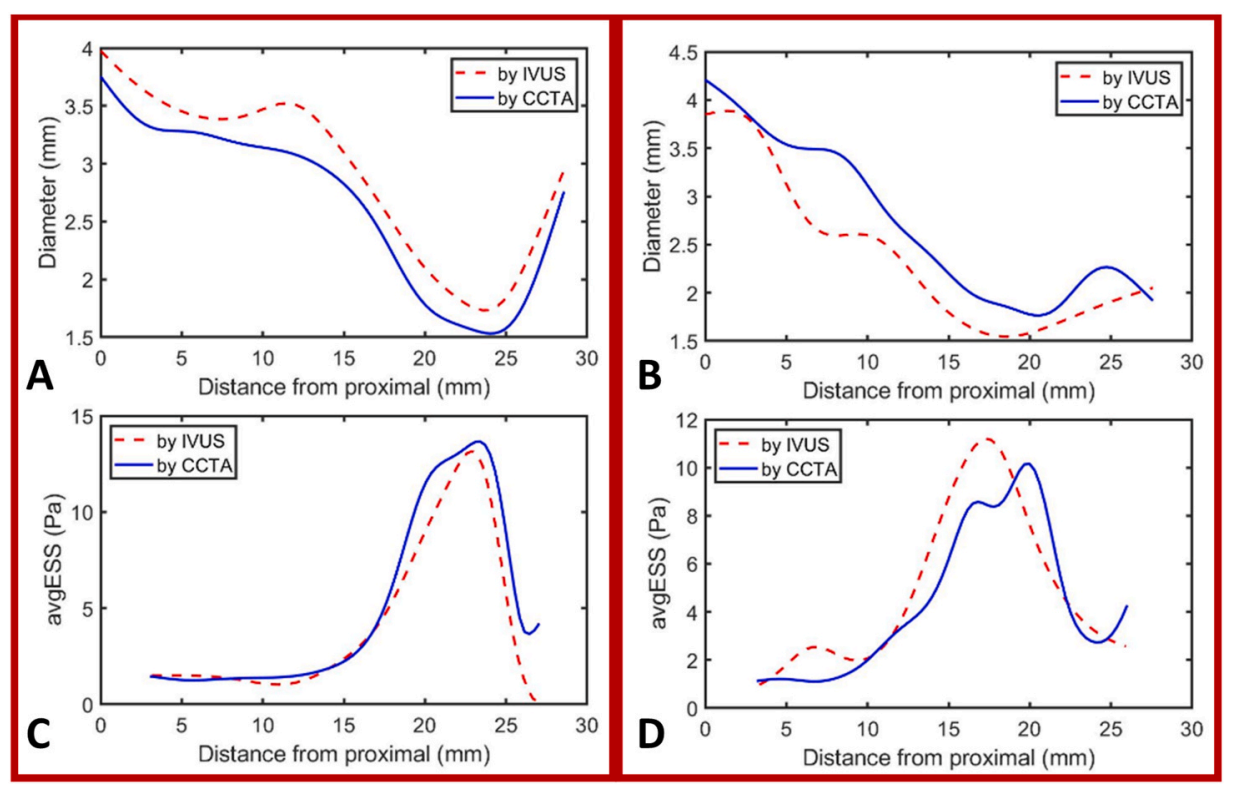

Fig. 6. IVUS vs CCTA for ESS evaluation along the course of coronary artery.

Graphical representation of diameter and endothelial shear stress (ESS) by both CCTA and IVUS. In ( $A$ and $C$ ) and ( $B$ and $D$ ) the same coronary vessel is represented. In (A and B) the lumen diameter is quantified by CCTA and IVUS with similar results, demonstrating a sudden reduction in lumen diameter at the same distance point from proximal, suggesting the presence of coronary stenosis. (C and D) Similarly, a punctual increase in ESS at the same distance point is well evident both for IVUS and CCTA, suggesting good accuracy for non-invasive evaluation of EES by CCTA. The present figure has been presented at the American Heart Association Congress 2019 as part of a poster presentation. 


\subsection{Limitations imaging plaque erosion}

The main limitation of the abovementioned techniques is the limited availability and cost. Moreover, definite diagnosis of plaque erosion relies on OCT, which entails an invasive evaluation with a dedicated catheter necessitating additional contrast injections. Among the noninvasive modalities, CCTA is the most widely used in clinical practice; nevertheless, CCTA has limited predictive capacity for plaque erosion and insufficient resolution to provide a diagnosis.

\subsection{Future perspectives}

Plaque erosion is the second most common causes of thrombus formation leading to acute coronary syndromes (ACS). Characterising this entity with an optimal imaging technique with high spatial and temporal resolution allows for tailoring treatment. Randomized studies are needed to confirm the best treatment strategy for patients with plaque erosion. Invasive plaque evaluation with OCT is the gold-standard for coronary plaque evaluation and should be pivotal in understanding the mechamism of ACS. Further studies are needed before non-invasive evaluation of plaque erosion could be applied to clinical practice. The development of molecular imaging combined with the anatomical visualization using invasive or non-invasive imaging techniques may hold interesting options for the future of plaque characterization. Moreover, these techniques may have the capacity to identify patients at risk of plaque erosion. Furthermore, the fusion imaging and blood flow simulation may have added value in stratifying patients at risk of coronary events.

\section{Declaration of competing interest}

The authors declared they do not have anything to disclose regarding conflict of interest with respect to this manuscript.

\section{References}

[1] P. Libby, G. Pasterkamp, F. Crea, et al., Reassessing the mechanisms of acute coronary syndromes, Circ. Res. 124 (2019) 150-160.

[2] T. Yonetsu, T. Kakuta, T. Lee, et al., In vivo critical fibrous cap thickness for rupture-prone coronary plaques assessed by optical coherence tomography, Eur. Heart J. 32 (2011) 1251-1259.

[3] J. Tian, X. Ren, R. Vergallo, et al., Distinct morphological features of ruptured culprit plaque for acute coronary events compared to those with silent rupture and thin-cap fibroatheroma: a combined optical coherence tomography and intravascular ultrasound study, J. Am. Coll. Cardiol. 63 (2014) 2209-2216.

[4] H. Jia, F. Abtahian, A.D. Aguirre, et al., In vivo diagnosis of plaque erosion and calcified nodule in patients with acute coronary syndrome by intravascular optical coherence tomography, J. Am. Coll. Cardiol. 62 (2013) 1748-1758.

[5] C.V. Bourantas, F.A. Jaffer, F.J. Gijsen, et al., Hybrid intravascular imaging: recent advances, technical considerations, and current applications in the study of plaque pathophysiology, Eur. Heart J. 38 (2017) 400-412.

[6] A. Maehara, M. Matsumura, Z.A. Ali, et al., IVUS-guided versus OCT-guided coronary stent implantation: a critical appraisal, JACC, Cardiovascular imaging 10 (2017) 1487-1503.

[7] R. Virmani, A.P. Burke, A. Farb, et al., Pathology of the vulnerable plaque, J. Am. Coll. Cardiol. 47 (2006) C13-C18.

[8] H. Jinnouchi, R. Virmani, A.V. Finn, Are characteristics of plaque erosion defined by optical coherence tomography similar to true erosion in pathology? Eur. Heart J. 39 (2018) 2086-2089.

[9] H. Sinclair, C. Bourantas, A. Bagnall, et al., OCT for the identification of vulnerable plaque in acute coronary syndrome, JACC. Cardiovascular imaging 8 (2015) 198-209.

[10] J. Dai, L. Xing, H. Jia, et al., In vivo predictors of plaque erosion in patients with ST-segment elevation myocardial infarction: a clinical, angiographical, and intravascular optical coherence tomography study, Eur. Heart J. 39 (2018) 2077-2085.

[11] H. Jia, J. Dai, J. Hou, et al., Effective anti-thrombotic therapy without stenting: intravascular optical coherence tomography-based management in plaque erosion (the EROSION study), Eur. Heart J. 38 (2017) 792-800.

[12] L. Xing, E. Yamamoto, T. Sugiyama, et al., EROSION study (effective antithrombotic therapy without stenting: intravascular optical coherence tomographybased management in plaque erosion): a 1-year follow-up report, Circulation. Cardiovascular interventions 10 (2017).

[13] J. Cuesta, P. Antuña, C. Jiménez, et al., Can plaque erosion Be visualized by highdefinition intravascular ultrasound? JACC Cardiovasc. Interv. 13 (2020) e57-e61.
[14] T. Higuma, T. Soeda, N. Abe, et al., A combined optical coherence tomography and intravascular ultrasound study on plaque rupture, plaque erosion, and calcified nodule in patients with ST-segment elevation myocardial infarction: incidence, morphologic characteristics, and outcomes after percutaneous coronary intervention, JACC Cardiovasc. Interv. 8 (2015) 1166-1176.

[15] J.E. Kwon, W.S. Lee, G.S. Mintz, et al., Multimodality intravascular imaging assessment of plaque erosion versus plaque rupture in patients with acute coronary syndrome, Korean Circ J 46 (2016) 499-506.

[16] I.D. Kilic, G. Caiazzo, E. Fabris, et al., Near-infrared spectroscopy-intravascular ultrasound: scientific basis and clinical applications, Eur Heart J Cardiovasc Imaging 16 (2015) 1299-1306.

[17] R. Waksman, C. Di Mario, R. Torguson, et al., Identification of patients and plaques vulnerable to future coronary events with near-infrared spectroscopy intravascular ultrasound imaging: a prospective, cohort study, Lancet (London, England) 394 (2019) 1629-1637.

[18] M. Yamaguchi, T. Sugiyama, M. Hoshino, et al., Two distinct phenotypes of plaque erosion assessed by multimodality intracoronary imaging: a case series, Eur Heart J Case Rep 4 (2020) 1-5.

[19] A.F. Stein-Merlob, T. Hara, J.R. McCarthy, et al., Atheroma susceptible to thrombosis exhibit impaired endothelial permeability in vivo as assessed by nanoparticle-based fluorescence molecular imaging, Circulation. Cardiovascular imaging 10 (2017).

[20] J. Knuuti, W. Wijns, A. Saraste, et al., 2019 ESC Guidelines for the diagnosis and management of chronic coronary syndromes: the Task Force for the diagnosis and management of chronic coronary syndromes of the European Society of Cardiology (ESC), Eur. Heart J. (2019).

[21] E. Conte, S. Mushtaq, G. Pontone, et al., Plaque quantification by coronary computed tomography angiography using intravascular ultrasound as a reference standard: a comparison between standard and last generation computed tomography scanners, Eur Heart J Cardiovasc Imaging 21 (2020) 191-201.

[22] C. Fischer, E. Hulten, P. Belur, et al., Coronary CT angiography versus intravascular ultrasound for estimation of coronary stenosis and atherosclerotic plaque burden: a meta-analysis, J Cardiovasc Comput Tomogr 7 (2013) 256-266.

[23] S. Voros, S. Rinehart, Z. Qian, et al., Coronary atherosclerosis imaging by coronary CT angiography: current status, correlation with intravascular interrogation and meta-analysis, JACC. Cardiovascular imaging 4 (2011) 537-548.

[24] P.D. Adamson, M.R. Dweck, D.E. Newby, The vulnerable atherosclerotic plaque: in vivo identification and potential therapeutic avenues, Heart 101 (2015) 1755-1766.

[25] E. Conte, A. Annoni, G. Pontone, et al., Evaluation of coronary plaque characteristics with coronary computed tomography angiography in patients with non-obstructive coronary artery disease: a long-term follow-up study, Eur Heart J Cardiovasc Imaging 18 (2017) 1170-1178.

[26] S. Motoyama, H. Ito, M. Sarai, et al., Plaque characterization by coronary computed tomography angiography and the likelihood of acute coronary events in mid-term follow-up, J. Am. Coll. Cardiol. 66 (2015) 337-346.

[27] P.D. Adamson, M.C. Williams, M.R. Dweck, et al., Guiding therapy by coronary CT angiography improves outcomes in patients with stable chest pain, J. Am. Coll. Cardiol. 74 (2019) 2058-2070.

[28] R. Erbel, S. Möhlenkamp, S. Moebus, et al., Coronary risk stratification, discrimination, and reclassification improvement based on quantification of subclinical coronary atherosclerosis: the Heinz Nixdorf Recall study, J. Am. Coll. Cardiol. 56 (2010) 1397-1406.

[29] M. Kashiwagi, A. Tanaka, H. Kitabata, et al., Feasibility of noninvasive assessment of thin-cap fibroatheroma by multidetector computed tomography, JACC Cardiovascular imaging 2 (2009) 1412-1419.

[30] S. Motoyama, M. Sarai, H. Harigaya, et al., Computed tomographic angiography characteristics of atherosclerotic plaques subsequently resulting in acute coronary syndrome, J. Am. Coll. Cardiol. 54 (2009) 49-57.

[31] Y. Ozaki, M. Okumura, T.F. Ismail, et al., Coronary CT angiographic characteristics of culprit lesions in acute coronary syndromes not related to plaque rupture as defined by optical coherence tomography and angioscopy, Eur. Heart J. 32 (2011) 2814-2823.

[32] I.J. van den Hoogen, U. Gianni, O. Al Hussein Alawamlh, et al., What atherosclerosis findings can CT see in sudden coronary death: plaque rupture versus plaque erosion, J Cardiovasc Comput Tomogr 14 (2020) 214-218.

[33] M.P. Opolski, A. Debski, J. Petryka, et al., CT for prediction of plaque erosion resulting in myocardial infarction with non-obstructive coronary arteries, J Cardiovasc Comput Tomogr 11 (2017) 237-239.

[34] D. Andreini, G. Pontone, S. Mushtaq, et al., Long-term prognostic impact of CTleaman score in patients with non-obstructive CAD: results from the COronary CT angiography Evaluation for clinical outcomes InteRnational multicenter (CONFIRM) study, Int. J. Cardiol. 231 (2017) 18-25.

[35] S.E. Lee, J.M. Sung, D. Andreini, et al., Differences in progression to obstructive lesions per high-risk plaque features and plaque volumes with CCTA, JACC, Cardiovascular imaging 13 (2020) 1409-1417.

[36] A. Ahmadi, E. Argulian, J. Leipsic, et al., From subclinical atherosclerosis to plaque progression and acute coronary events: JACC state-of-the-art review, J. Am. Coll. Cardiol. 74 (2019) 1608-1617.

[37] E. Yamamoto, V. Thondapu, E. Poon, et al., Endothelial shear stress and plaque erosion: a computational fluid dynamics and optical coherence tomography study, JACC. Cardiovascular imaging 12 (2019) 374-375.

[38] P.H. Stone, A. Maehara, A.U. Coskun, et al., Role of low endothelial shear stress and plaque characteristics in the prediction of nonculprit major adverse cardiac events: the PROSPECT study, JACC. Cardiovascular imaging 11 (2018) 462-471.

[39] H. Samady, D.S. Molony, A.U. Coskun, et al., Risk stratification of coronary plaques using physiologic characteristics by CCTA: focus on shear stress, J Cardiovasc Comput Tomogr (2019). 
[40] J.B. Park, G. Choi, E.J. Chun, et al., Computational fluid dynamic measures of wall shear stress are related to coronary lesion characteristics, Heart 102 (2016) $1655-1661$.

[41] J.M. Lee, G. Choi, B.K. Koo, et al., Identification of High-Risk Plaques Destined to Cause Acute Coronary Syndrome Using Coronary Computed Tomographic Angiography and Computational Fluid Dynamics, JACC, 2018. Cardiovascular imaging.

[42] D. Han, A. Starikov, B. Oh, et al., Relationship between endothelial wall shear stress and high-risk atherosclerotic plaque characteristics for identification of coronary lesions that cause ischemia: a direct comparison with fractional flow reserve, Journal of the American Heart Association 5 (2016).

[43] C. Costopoulos, L.H. Timmins, Y. Huang, et al., Impact of combined plaque structural stress and wall shear stress on coronary plaque progression, regression, and changes in composition, Eur. Heart J. 40 (2019) 1411-1422.
[44] T. Hoshi, A. Sato, D. Akiyama, et al., Coronary high-intensity plaque on T1weighted magnetic resonance imaging and its association with myocardial injury after percutaneous coronary intervention, Eur. Heart J. 36 (2015) 1913-1922.

[45] M.Y. Henein, S. Vancheri, G. Bajraktari, et al., Coronary Atherosclerosis Imaging, Diagnostics (Basel), vol. 10, 2020.

[46] S. Kato, K. Kitagawa, N. Ishida, et al., Assessment of coronary artery disease using magnetic resonance coronary angiography: a national multicenter trial, J. Am. Coll. Cardiol. 56 (2010) 983-991.

[47] M. Kolossváry, J. Park, J.I. Bang, et al., Identification of invasive and radionuclide imaging markers of coronary plaque vulnerability using radiomic analysis of coronary computed tomography angiography, Eur Heart J Cardiovasc Imaging 20 (2019) 1250-1258. 